\title{
ON THE HARDNESS OF APPROXIMATING SOME NP-OPTIMIZATION PROBLEMS RELATED TO MINIMUM LINEAR ORDERING PROBLEM
}

\author{
SOUNAKA Mishra ${ }^{1}$ AND KRIPASINDHU SIKDAR ${ }^{1}$
}

\begin{abstract}
We study hardness of approximating several minimaximal and maximinimal NP-optimization problems related to the minimum linear ordering problem (MINLOP). MINLOP is to find a minimum weight acyclic tournament in a given arc-weighted complete digraph. MINLOP is APX-hard but its unweighted version is polynomial time solvable. We prove that MIN-MAX-SUBDAG problem, which is a generalization of MINLOP and requires to find a minimum cardinality maximal acyclic subdigraph of a given digraph, is, however, APX-hard. Using results of Håstad concerning hardness of approximating independence number of a graph we then prove similar results concerning MAX-MIN-VC (respectively, MAX-MIN-FVS) which requires to find a maximum cardinality minimal vertex cover in a given graph (respectively, a maximum cardinality minimal feedback vertex set in a given digraph). We also prove APX-hardness of these and several related problems on various degree bounded graphs and digraphs.
\end{abstract}

Mathematics Subject Classification. 68Q17, 68R01, 68W25.

\section{INTRODUCTION}

In this paper we deal with hardness of approximating several minimum-maximal (or simply, minimaximal) and maximum-minimal (or simply, maximinimal) NPcomplete optimization problems on graphs as well as related maximum/minimum problems. In general, for any given instance $x$ of such a problem, it is required to find a minimum (respectively, maximum) weight (or, cardinality) maximal

Keywords and phrases: NP-optimization problems, Minimaximal and maximinimal NP-optimization problems, Approximation algorithms, Hardness of approximation, APX-hardness, AP-reduction, L-reduction, S-reduction.

1 Theoretical Statistics and Mathematics Unit, Indian Statistical Institute, Calcutta 700035 , India; e-mail: res9513@isical.ac.in, sikdar@isical.ac.in 
(respectively, minimal) feasible solution with respect to a partial order on the set of feasible solutions of $x$. The terminology of minimaximal and maximinimal is apparently first used by Peters et al. [29], though the concept has received attention of many others, specially in connection with many graph problems. We may cite for example, minimum chromatic number and its maximum version, the achromatic number $[9,12,20,21]$, maximum independent set and minimaximal independent set (minimum independent dominating set) [18, 22, 23, 25], minimum vertex cover and maximinimal vertex cover $[28,30]$, minimum dominating set and maximinimal dominating set [10,27], minimum vertex (edge) connectivity and maximinimal vertex (edge) connectivity [20,29] and a recent systematic study of minimaximal and maximinimal optimization problems by Manlove [30].

We are led to investigation of several such graph problems while considering a generalization of the minimum linear ordering problem (MINLOP). Given a complete digraph $G_{n}=\left(V, A_{n}\right)$ on a set $V=\left\{v_{1}, v_{2}, \ldots v_{n}\right\}$ of $n$ vertices with nonnegative integral arc weights, the MINLOP is to find an acyclic tournament [19] on $V$ with minimum total arc weight. This is a known NP-complete optimization problem $[7,16,17,31]$. In [31] it is shown that MINLOP is APX-hard, but $\triangle_{t}$-MINLOP (i.e., MINLOP satisfying a parameterized triangle inequality with parameter $t \in(0,2])$ is $\frac{2+t}{2 t}$-approximable, $\triangle_{2}$-MINLOP is in PO, and, for any $t \in(0,2), \triangle_{t}$-MINLOP does not admit a polynomial time approximation scheme, unless $P=$ NP. Two problems related to MINLOP are the maximum acyclic subdigraph (MAX-SUBDAG) and the minimum feedback arc set (MIN-FAS) problems. Given a digraph $G=(V, A)$, the MAX-SUBDAG (respectively, MIN-FAS) problem is to find a subset of $B \subseteq A$ of maximum (respectively, minimum) cardinality such that $(V, B)$ (respectively, $(V, A-B)$ ) is an acyclic subdigraph (SUBDAG) of $G$. While MAX-SUBDAG is APX-complete [28] and has a trivial 2-approximate algorithm, MINFAS is not known to be in APX, though it is APX-hard [24].

A generalization of MINLOP can be formulated as follows. Note that an acyclic tournament on $V$ is indeed a maximal SUBDAG of $G_{n}$ (i.e., a SUBDAG of $G_{n}$ which is not contained in any SUBDAG of $G_{n}$ ). Thus we generalize MINLOP as the minimum weight maximal SUBDAG (MIN-W-MAX-SUBDAG) problem which requires to find a maximal SUBDAG of minimum total arc weight in any given arc weighted digraph (which is not necessarily a complete digraph). MIN-W-MAXSUBDAG is thus APX-hard as its special case MINLOP is so. For MIN-W-MAXSUBDAG we can not talk about arc weights satisfying triangle (or parameterized triangle) inequality, and so there is no immediate answer to the question whether it is in APX with suitable restrictions on arc weights. But it appears to be unlikely as we show that unweighted version (i.e., all arc weights 1) of MIN-W-MAXSUBDAG, called MIN-MAX-SUBDAG, is APX-hard even though MINLOP with constant arc weight is solvable in polynomial time.

The complementary problem of MIN-MAX-SUBDAG is the maximum cardinality minimal feedback arc set (MAX-MIN-FAS) in which it is required to find a minimal feedback arc set of maximum cardinality in a given digraph. The vertex version of this is the maximum cardinality minimal feedback vertex set 
(MAX-MIN-FVS). An NP-optimization problem related to MAX-MIN-FVS is MAX-MIN-VC, in which it is required to find a minimal vertex cover of maximum cardinality in a given graph. The related problem of minimum cardinality maximal independent set (MIN-MAX-IS) problem, where one is required to find a maximal IS (or an independent dominating set) of minimum cardinality for any given graph. MIN-MAX-IS and MAX-MIN-VC have the same complexity over every graph class ([30], Th. 4.2.11) though they may not have similar approximation properties and are NP-complete even for bipartite graphs $[22,23]$ and dually chordal graphs [5] though polynomial time algorithms are known for many special classes of graphs such as chordal graphs [13], interval and circular-arc graphs [8], permutation graphs $[6,14]$ and many other graph classes (see [30]).

Since the decision versions of these optimization problems are NP-complete, it is not possible to find optimal solutions in polynomial time, unless $P=$ NP. So a practical alternative is to find near optimal (or approximate) solutions in polynomial time. However, it is not always possible to obtain such solutions having desired approximation properties $[2,18,26,28]$. Thus, it is of considerable theoretical and practical interest to provide some qualitative explanation for this by establishing results about hardness of obtaining such approximate solutions.

In this paper, we shall establish several results about hardness of approximating such problems using the standard technique of reduction of one problem to another. The paper is organized as follows. In Section 2, we recall the relevant concepts about graphs, digraphs, NP-optimization problems and approximation algorithms. In Section 3, we first prove APX-hardness of MIN-MAX-SUBDAG for arbitrary digraph by reducing MAX-SUBDAG to it. Then, using the results of Håstad concerning hardness of approximating MAX-IS, we prove similar results about MAX-MIN-VC for arbitrary graphs and about MAX-MIN-FVS for arbitrary graphs and digraphs. In Section 4, we show that, MIN-FVS is APXcomplete for 6-regular graphs and MAX-MIN-FVS is APX-hard for all graphs of maximum degree 9 . We also prove that MAX-MIN-VC is $k$-approximable for all graphs without any isolated vertex and having maximum degree $k, k \geq 1$, and is APX-complete for all graphs of maximum degree 5. In Section 5, we prove APX-hardness of MIN-FAS and MAX-SUBDAG for $k$-total-regular digraphs, for all $k \geq 4$. Then we show that MIN-MAX-SUBDAG is APX-hard for digraphs of maximum total degree 12 and MAX-MIN-FVS is APX-hard for all digraphs of maximum total degree 6. Finally, in Section 6, we make some concluding remarks.

\section{BASIC CONCEPTS}

We will denote a graph (i.e. an undirected graph) by $G=(V, E)$ and a digraph (i.e. a directed graph) by $G=(V, A)$, where $V=\left\{v_{1}, v_{2}, \ldots v_{n}\right\}, E$ is the edge set and $A$ is the arc set. An edge between vertices $v_{i}$ and $v_{j}$ is an unordered pair which will be denoted by $\left\{v_{i}, v_{j}\right\}$, whereas an arc from $v_{i}$ to $v_{j}$ will be denoted by the ordered pair $\left(v_{i}, v_{j}\right)$. If $\left\{v_{i}, v_{j}\right\}$ is an edge in $G$ then we say that it is incident on $v_{i}$ and $v_{j}$. If $\left(v_{i}, v_{j}\right)$ is an arc in $G$ then $v_{i}$ is the initial point and $v_{j}$ 
is the terminal point of $\left(v_{i}, v_{j}\right)$. In an undirected graph $G$, degree of a vertex $v_{i}$ is denoted as $d\left(v_{i}\right)$ which is the number of edges incident on $v_{i}$ in $G$, and $G$ is called $k$-regular if each vertex in $G$ has degree $k$. In a digraph $G, d^{+}\left(v_{i}\right)$ and $d^{-}\left(v_{i}\right)$ are the number of arcs in $G$ having $v_{i}$ as the initial vertex and terminal vertex, respectively, and $d\left(v_{i}\right)$, the total degree of $v_{i}$ is defined as $d\left(v_{i}\right)=d^{+}\left(v_{i}\right)+d^{-}\left(v_{i}\right)$. A digraph $G$ is $k$-total-regular if for each vertex $v_{i}, d\left(v_{i}\right)=k$. A path $P\left(v_{1}, v_{t}\right)$ in $G=(V, E)$ (respectively, dipath in $G=(V, A))$ is a sequence of distinct vertices $\left(v_{1}, v_{2}, \ldots, v_{t}\right)$ such that $\left\{v_{i}, v_{i+1}\right\} \in E$ (respectively, $\left(v_{i}, v_{i+1}\right) \in A$ ) for $1 \leq i<t$. A path (respectively, dipath) $P\left(v_{1}, v_{t}\right)$ is called a cycle (respectively, dicycle) if $v_{1}=v_{t}$.

A tournament on $V[19]$ is a digraph $G=(V, T)$ such that, for every two vertices $u, v \in V, T$ contains exactly one arc with end vertices $u$ and $v$. A tournament on $V$ is called an acyclic tournament on $V$ if it does not contain any directed cycle. An feedback arc set (FAS) in a digraph $G=(V, A)$ is an arc set $B \subseteq A$ such that the subdigraph $(V, A-B)$ is acyclic. Given a digraph $G=(V, A)$, a minimal $F A S$ is an FAS $B \subseteq A$ which does not contain another FAS. $(V, B)$ is called a directed acyclic subgraph (SUBDAG) of $G=(V, A)$ if $B \subseteq A$ and has no directed cycle. A SUBDAG $(V, B)$ of $G$ is called a maximal SUBDAG if there exists no SUBDAG $\left(V, B^{\prime}\right)$ of $G$ such that $B \subset B^{\prime}$. Given a graph $G=(V, E), C \subseteq V$ is called a vertex cover (VC) if for each edge $\left\{v_{i}, v_{j}\right\} \in E, C$ contains either $v_{i}$ or $v_{j}$. A VC $C$ is called a minimal $V C$ of $G$ if no proper subset of $C$ is also a VC of $G$. $S \subseteq V$ is called an feedback vertex set (FVS) of $G$ if the subgraph/subdigraph $G[V-S]$ induced by the vertex set $V-S$ is acyclic. Similarly a minimal $F V S$ of $G$ is defined.

For definitions and notations concerning basic concepts regarding approximation algorithms for NP-optimization problems we refer to the book [3].

The precise formulations of some of the problems we deal with are given below where we have ommited the goal as it can be inferred from the name of the problem.

\section{MINLOP}

Instance - A pair $x=\left(G_{n}, w\right)$, where $G_{n}=\left(V, A_{n}\right)$ is a complete digraph on $V$ and $w$ assigns a nonnegative integer to any $e \in A_{n}$.

Solution - An acyclic tournament $y$ on $V$.

Cost $-m(x, y)=\max \left\{1, \sum_{e \in y} w(e)\right\}$.

MIN-VC

Instance - A graph $x=G=(V, E)$.

Solution - A VC $C$ of $G$.

Cost $-m(x, C)=\max \{1,|C|\}$.

MAX-W-SUBDAG

Instance - A pair $x=(G, w)$, where $G=(V, A)$ is a digraph and $w$ assigns a nonnegative integer to each $e \in A$.

Solution - A SUBDAG $(V, B)$ of $G$.

Cost $-m(x, B)=\max \left\{1, \sum_{e \in B} w(e)\right\}$. 


\section{MIN-W-FAS}

Instance - A pair $x=(G, w)$, where $G=(V, A)$ is a digraph and $w$ assigns a nonnegative integer to each $e \in A$.

Solution - An FAS $B$ of $G$.

Cost $-m(x, B)=\max \left\{1, \sum_{e \in B} w(e)\right\}$.

\section{MIN-W-FVS}

Instance - A pair $x=(G, w)$ where $G$ is a graph/digraph and $w$ assigns a nonnegative integer to each $v \in V$.

Solution - An FVS $F$ of $G$.

Cost $-m(x, F)=\max \left\{1, \sum_{v \in F} w(v)\right\}$.

In an unweighted version of a weighted optimization problem, we assume that weight function is a constant function and this constant is 1 . We denote the unweighted problem without the letter "W" in the problem name. For a given graph parameter $P$ (for example, the parameter $P$ is vertex cover), in the optimization problem MIN- $P$, it is required to find a minimum weight solution over all minimal solutions. In the corresponding MAX-MIN- $P$ problem, it is required to find a maximum weight minimal solution over all minimal solutions satisfying the parameter $P$. For example, in MAX-MIN-VC, it is required to find a minimal vertex cover of maximum weight in a given vertex weighted graph $(G, w)$. Similarly, we define MAX-MIN-FAS, MAX-MIN-FVS and MIN-MAX-SUBDAG. In a graph optimization problem $\pi$, if the input graph (or, digraph) is $k$-regular (or, $k$-totalregular) then we denote this restricted problem as $\pi-k$; and if the input graph (or, digraph) is of degree (or, total-degree) atmost $k$ then we denote this restricted problem as $\pi-\leq k$. Other NP-optimization problems that we consider are MINMAX-SUBDAG, MIN-MAX-SUBDAG- $\leq k$, MAX-MIN-VC, MAX-MIN-VC- $\leq k$, MAX-MIN-FVS, MAX-MIN-FVS- $\leq k$, MIN-W-FAS- $\leq k$, MAX-W-SUBDAG- $\leq k$ and MIN-FVS-6.

We use the performance ratio [3] $R_{\pi}(x, y)$ as a measure of quality of an approximate solution $y$ of an instance $x$ of $\pi$. Various notations of approximate algorithms and approximation classes such as APX, PTAS, FPTAS are formulated as in [3] in terms of $R_{\pi}(x, y)$. Though the completeness concepts in approximation classes are defined with respect to AP-reduction [11], we shall use $L$-reduction [28] which suffices our purpose due to the following theorem.

Theorem 2.1. [3] For any two NPO problems $\pi_{1}$ and $\pi_{2}$, if $\pi_{1} \leq_{L} \pi_{2}$ and $\pi_{1} \in A P X$, then $\pi_{1} \leq_{A P} \pi_{2}$.

One purpose of a reduction from $\pi_{1}$ to $\pi_{2}$ is to use an approximation algorithm for $\pi_{2}$ to construct an equally good approximation algorithm for $\pi_{1}$. The approximation preserving reductions, for example $L$-reduction, work very well when reducing to the problems with bounded approximation, but this is not the case when reducing to the problems that can not be approximated within a constant. This is because, these reductions may transform an input instance of $\pi_{1}$ to a much larger input instance of $\pi_{2}$. Because of the size amplification the constructed algorithm for $\pi_{1}$ via a reduction may not be as good as the original algorithm for $\pi_{2}$. 
Kann [25] introduced a size dependent reduction (called $S$-reduction) between problems that can not be approximated within a constant.

$\pi_{1}$ is said to be $S$-reducible to $\pi_{2}$, in symbol $\pi_{1} \leq_{S} \pi_{2}$, with size amplification $\alpha(n)$ from $\pi_{1}$ to $\pi_{2}$ is a tuple $(f, g, \alpha, c)$ such that:

1. $f$ and $g$ are polynomial time computable functions, $\alpha$ is a monotone increasing positive function and $c$ is a positive constant;

2. $f: I_{\pi_{1}} \rightarrow I_{\pi_{2}}$ and $\forall y \in \operatorname{sol}_{\pi_{2}}(f(x)), g(x, y) \in \operatorname{sol}_{\pi_{1}}(x)$;

3. $\forall x \in I_{\pi_{1}}$ and $\forall y \in \operatorname{sol}_{\pi_{2}}(f(x)), R_{\pi_{1}}(x, g(x, y)) \leq c \cdot R_{\pi_{2}}(f(x), y)$;

4. $\forall x \in I_{\pi_{1}},|f(x)| \leq \alpha(|x|)$.

The following theorem follows from the definition of $S$-reduction.

Theorem 2.2. [25] Given two NPO problems $\pi_{1}$ and $\pi_{2}$, if $\pi_{1} \leq_{S} \pi_{2}$ with size amplification $\alpha(n)$ and $\pi_{2}$ can be approximated within some monotone increasing positive function $u(n)$ of the size of the input instance, then $\pi_{1}$ can be approximated within $c \cdot u(\alpha(n))$, which is a monotone increasing positive function.

The following theorem says how to get lower bound about approximability of $\pi_{2}$ from the known lower bound of $\pi_{1}$ by using a $S$-reduction from $\pi_{1}$ to $\pi_{2}$.

Theorem 2.3. Let $\pi_{1} \leq_{S} \pi_{2}$ with size amplification $\alpha(n)$. Let $n=|x| \in I_{\pi_{1}}$ and $N=|f(x)| \leq a n^{k}$, for $n \geq n_{0}$, where $a \geq 1, k>0$ and $n_{0}$ is a positive integer. If there exists no polynomial time algorithm to approximate $\pi_{1}$ within a factor of $\frac{1}{p} n^{q-\epsilon}$ for any $\epsilon>0$, for some positive constants $p$ and $q$, unless $P=\mathrm{NP}$ (unless $\mathrm{NP}=\mathrm{ZPP})$; then there exists no polynomial time algorithm to approximate $\pi_{2}$ within a factor of $\frac{1}{p_{c a}^{q / k}} N^{\frac{q}{k}-\epsilon}$, for any $\epsilon>0$, unless $P=\mathrm{NP}$ (unless $\mathrm{NP}=\mathrm{ZPP}$ ).

Proof. Proof is quite trivial and depends on the inequality $\frac{1}{p c} n^{q-\epsilon} \geq \frac{1}{p c a^{q / k}} N^{\frac{q}{k}-\frac{\epsilon}{k}}$, which follows from $N \leq a n^{k}$ and $a \geq 1$.

To conclude this section, we state two results due to Håstad which we shall use in Section 3 for proving similar results for MAX-MIN-VC and MAX-MIN-FVS.

Theorem 2.4. [18] Unless $\mathrm{NP}=\mathrm{ZPP}$, for any $\epsilon>0$ there exists no polynomial time algorithm to approximate $M A X-I S$ within a factor of $n^{1-\epsilon}$, where $n$ is the number of vertices in an instance.

Theorem 2.5. [18] Unless $P=\mathrm{NP}$, for any $\epsilon>0$ there exists no polynomial time algorithm to approximate MAX-IS within a factor of $n^{\frac{1}{2}-\epsilon}$, where $n$ is the number of vertices in an instance.

\section{HARDNESS RESUlTS FOR ARBITRARY GRAPHS/DIGRAPHS}

In this section, we prove hardness results for several minimaximal and maximinimal problems for general graphs and digraphs. First we consider MIN-MAXSUBDAG problem. As already noted, MIN-W-MAX-SUBDAG is APX-hard as it is a generalization of MINLOP which is APX-hard [31]. However, even though MINLOP can be solved in polynomial time when the arc weights are constant, as we show in Theorem 3.1, MIN-MAX-SUBDAG is APX-hard. 
Theorem 3.1. MIN-MAX-SUBDAG is APX-hard.

Proof. It is enough to show that MAX-SUBDAG $\leq_{L}$ MIN-MAX-SUBDAG as MAXSUBDAG is APX-complete [28].

For each instance $x=(G=(V, A))$ of MAX-SUBDAG, we construct in polynomial time an instance $f(x)=\left(G^{\prime}=\left(V^{\prime}, A^{\prime}\right)\right)$ of MIN-MAX-SUBDAG and with each feasible solution $S^{\prime}$ of $f(x)$, we associate a feasible solution $g\left(S^{\prime}\right)=S=S^{\prime} \cap A$ of $x$ such that $f$ and $g$ satisfy the conditions of $L$-reduction with $\alpha=5$ and $\beta=1$.

Let $K=\left\{\left(v_{i}, v_{j}\right) \mid\left(v_{i}, v_{j}\right) \in A\right.$ and $\left.\left(v_{j}, v_{i}\right) \notin A\right\}$. For each $\operatorname{arc}\left(v_{i}, v_{j}\right) \in K$, we introduce a new vertex $v_{i j}$ for the construction of $G^{\prime}$. Construct $G^{\prime}=\left(V^{\prime}, A^{\prime}\right)$ as follows: $V^{\prime}=V \cup\left\{v_{i j} \mid\left(v_{i}, v_{j}\right) \in K\right\}$ and $A^{\prime}=A \cup\left\{\left(v_{j}, v_{i}\right),\left(v_{j}, v_{i j}\right),\left(v_{i j}, v_{i}\right) \mid\left(v_{i}, v_{j}\right) \in\right.$ $K\}$. For an example, see Figure 1. Let $k=|K|$ and $p$ be the number of pairs of vertices $v_{i}, v_{j} \in V$ such that both $\left(v_{i}, v_{j}\right),\left(v_{j}, v_{i}\right) \in A$. Hence, $p=\frac{|A-K|}{2}$.

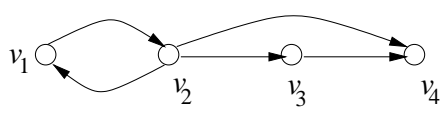

$G$

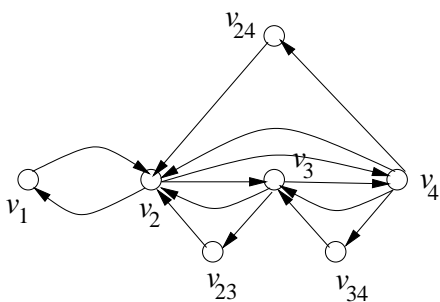

$G^{\prime}$

Figure 1. A digraph $G$ and the corresponding digraph $G^{\prime}$.

Next we establish a few claims.

Claim 3.2. Let $\left(V^{\prime}, S^{\prime}\right)$ be a maximal SUBDAG of $G^{\prime}$ and $S=S^{\prime} \cap A$. Then $(V, S)$ is a SUBDAG of $G$ and $\left|S^{\prime}\right|=3 k+2 p-|S|$.

Proof. Since $(V, S)$ is just a subdigraph of $\left(V^{\prime}, S^{\prime}\right)$, it is acyclic and so $(V, S)$ is a SUBDAG of $G$.

Now note that an arc in $S^{\prime}-A$ must come from the set $\left\{\left(v_{j}, v_{i}\right),\left(v_{j}, v_{i j}\right),\left(v_{i j}, v_{i}\right)\right\}$ for any $\operatorname{arc}\left(v_{i}, v_{j}\right) \in K$. As $\left(V^{\prime}, S^{\prime}\right)$ is a maximal SUBDAG of $G^{\prime}$, if $\left(v_{i}, v_{j}\right) \in$ $K \cap S^{\prime}$, then $S^{\prime}$ must contain exactly one of $\left(v_{j}, v_{i j}\right)$ and $\left(v_{i j}, v_{i}\right)$, and if $\left(v_{i}, v_{j}\right) \in$ $K-S^{\prime}$, then $S^{\prime}$ must contain all the three $\operatorname{arcs}\left(v_{j}, v_{i}\right),\left(v_{j}, v_{i j}\right)$ and $\left(v_{i j}, v_{i}\right)$. So, $\left|S^{\prime}\right|=|S|+k+2|K-S|$. We now show that $|K-S|=k+p-|S|$. Note that $|K-S|=|K|-|K \cap S|$ and $|K \cap S|=|S|-|S-K|$. Also $S-K=\left(A \cap S^{\prime}\right)-K=$ $(A-K) \cap S^{\prime}$. Now $S^{\prime}$ contains exactly one of the two $\left(v_{i}, v_{j}\right)$ and $\left(v_{j}, v_{i}\right)$ for every pair of $\operatorname{arcs}\left(v_{i}, v_{j}\right)$ and $\left(v_{j}, v_{i}\right)$ in $A-K$ as $\left(V^{\prime}, S^{\prime}\right)$ is a maximal SUBDAG of $G^{\prime}$. It follows that $\left|(A-K) \cap S^{\prime}\right|=\frac{1}{2}|A-K|=p$ and $|S-K|=p$ so $|K \cap S|=|S|-p$. Thus $\left|S^{\prime}\right|=|S|+k+2(k+p-|S|)=3 k+2 p-|S|$.

Claim 3.3. If $\left(V^{\prime}, S_{o}^{\prime}\right)$ is a minimum maximal SUBDAG of $G^{\prime}$, then $\left(V, S_{o}=\right.$ $\left.S_{o}^{\prime} \cap A\right)$ is a maximum SUBDAG of $G$. Also $|A| \leq 2\left|S_{o}\right|$. 
Proof. If $\left(V, S_{o}\right)$ is not a maximum SUBDAG of $G$, then let $(V, \tilde{S})$ be a maximum SUBDAG of $G$. Let $\tilde{S}^{\prime}=\tilde{S} \cup\left\{\left(v_{i j}, v_{i}\right) \mid\left(v_{i}, v_{j}\right) \in K \cap \tilde{S}\right\} \cup\left\{\left(v_{j}, v_{i}\right),\left(v_{j}, v_{i j}\right),\left(v_{i j}, v_{i}\right) \mid\right.$ $\left.\left(v_{i}, v_{j}\right) \in K-\tilde{S}\right\}$. Now clearly $\tilde{S}=\tilde{S}^{\prime} \cap A$. Also $\left(V, \tilde{S}^{\prime}\right)$ is a maximal SUBDAG of $G^{\prime}$. This is because:

(a) as $(V, \tilde{S})$ is a maximum SUBDAG of $G$ and $\tilde{S}^{\prime}$ contains $\tilde{S}$, no arc from $A-\tilde{S}$ can be added to $\tilde{S}^{\prime}$ without creating a dicycle, and

(b) no arc from $A^{\prime}-\left(A \cup \tilde{S}^{\prime}\right)$ can be added to $\tilde{S}^{\prime}$ without creating a dicycle. To see this, note that $A^{\prime}-\left(A \cup \tilde{S}^{\prime}\right)=\left\{\left(v_{j}, v_{i}\right),\left(v_{j}, v_{i j}\right) \mid\left(v_{i}, v_{j}\right) \in K \cap \tilde{S}\right\}$. As both $\left(v_{i}, v_{j}\right)$ and $\left(v_{i j}, v_{i}\right) \in \tilde{S}^{\prime}$, we can not add any arc from $A^{\prime}-\left(A \cup \tilde{S}^{\prime}\right)$ to $\tilde{S}^{\prime}$ without creating a dicycle.

Also by Claim 3.2, $\left|\tilde{S}^{\prime}\right|=3 k+2 p-|\tilde{S}|$. Since $|\tilde{S}|>\left|S_{o}\right|, 3 k+2 p-\left|S_{o}\right|=$ $\left|S_{o}^{\prime}\right|>3 k+2 p-|\tilde{S}|$, which contradicts the assumption that $\left(V^{\prime}, S_{o}^{\prime}\right)$ is a minimum cardinality maximal SUBDAG of $G^{\prime}$.

To show that $|A| \leq 2\left|S_{o}\right|$, it is enough to observe that as $\left(V, S_{o}\right)$ is a maximum SUBDAG of $G,\left(V, A-S_{o}\right)$ is a SUBDAG of $G$. Hence, $\left|S_{o}\right| \geq\left|A-S_{o}\right|=|A|-\left|S_{o}\right|$, i.e., $2\left|S_{o}\right| \geq|A|$.

Now returning to the proof of Theorem 3.1, note that $\left|S_{o}^{\prime}\right|=3 k+2 p-\left|S_{o}\right| \leq$ $3(k+p)-\left|S_{o}\right| \leq 6\left|S_{o}\right|-\left|S_{o}\right|=5\left|S_{o}\right|$. Also for any maximal SUBDAG $\left(V^{\prime}, S^{\prime}\right)$ of $G^{\prime},\left|S_{o}\right|-|S|=\left|S^{\prime}\right|-\left|S_{o}^{\prime}\right|$.

Next we prove results about hardness of approximating MAX-MIN-VC and MAX-MIN-FVS, using $S$-reducibility arguments and the results of Håstad concerning MAX-IS, Theorem 2.4 and Theorem 2.5.

Theorem 3.4. Unless NP $=\mathrm{ZPP}$, for any $\epsilon>0$ there exists no polynomial time algorithm to approximate MAX-MIN-VC within a factor of $\frac{1}{2 \sqrt{2}} n^{\frac{1}{2}-\epsilon}$, where $n$ is the number of vertices in an instance.

Proof. Given an instance $G=(V, E)$ of MAX-IS, we construct an instance $G^{\prime}=$ $\left(V^{\prime}, E^{\prime}\right)$ of MAX-MIN-VC, where $V^{\prime}=V \cup\left[\cup_{v \in V}\left\{v^{1}, v^{2}, \ldots v^{n+1}\right\}\right]$ and $E^{\prime}=$ $E \cup\left\{\left\{v, v^{1}\right\},\left\{v, v^{2}\right\}, \ldots\left\{v, v^{n+1}\right\} \mid v \in V\right\}$. In other words, $G^{\prime}$ is obtained from $G$ by introducing for each vertex $v \in V, n+1$ additional vertices $v^{1}, v^{2}, \ldots, v^{n+1}$ and $(n+1)$ additional edges $\left\{v, v^{1}\right\},\left\{v, v^{2}\right\}, \ldots\left\{v, v^{n+1}\right\}$ to the graph $G$ (for example see Fig. 2).

We first establish a few claims.

Claim 3.5. A vertex cover $S^{\prime} \subseteq V^{\prime}$ of $G^{\prime}$ is a minimal VC iff

(a) for $v \in S^{\prime} \cap V, v^{i} \notin S^{\prime}$, for any $1 \leq i \leq n+1$, and

(b) for $v \in V-S^{\prime},\left\{v^{1}, v^{2}, \ldots v^{n+1}\right\} \subseteq S^{\prime}$.

Proof. Let $S^{\prime}$ be a minimal $\mathrm{VC}$ of $G^{\prime}$. If $v \in S^{\prime} \cap V$, then for $1 \leq i \leq n+1$, no $v^{i}$ is in $S^{\prime}$ as $S^{\prime}$ is a minimal $\mathrm{VC}$ and $v$ covers the edge $\left\{v, v^{i}\right\}$. If $v \in V-S^{\prime}$, then to cover the edges $\left\{v, v^{i}\right\}, 1 \leq i \leq n+1, S^{\prime}$ must include $v^{i}$.

Conversely, suppose $S^{\prime}$ satisfies (a) and (b) of Claim 3.5. We will show that we can not drop any vertex from $S^{\prime}$ to get a proper subset $S^{\prime \prime} \subset S^{\prime}$ which is also a $\mathrm{VC}$ of $G^{\prime}$. If $v \in S^{\prime} \cap V$ then, as for $1 \leq i \leq n+1$, none of $v^{i}$ is in $S^{\prime}$ and none 


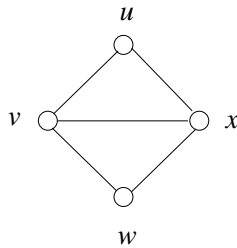

$G=(V, E)$

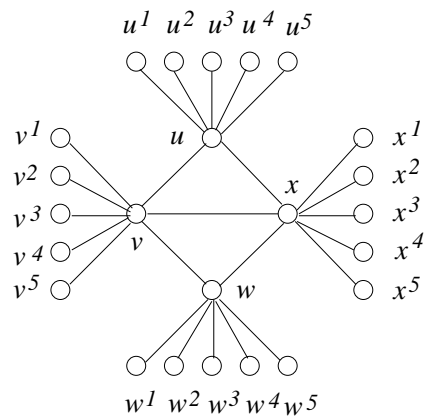

$G^{\prime}=\left(V^{\prime}, E^{\prime}\right)$

Figure 2. An instance $G$ of MAX-IS and the corresponding instance $G^{\prime}$ of MAX-MIN-VC.

of the edges $\left\{v, v^{i}\right\}$ can be covered by a vertex in $S^{\prime}$ other than $v, v$ can not be dropped from $S^{\prime}$. If $v \in V-S^{\prime}$, then no vertex $v^{i} \in S^{\prime}, 1 \leq i \leq n+1$, can be dropped from $S^{\prime}$, as no vertex in $S^{\prime}-\left\{v^{i}\right\}$ will cover the edge $\left\{v, v^{i}\right\}$.

From the Claim 3.5 it follows that, for any minimal VC $S^{\prime} \subseteq V^{\prime}$ of $G^{\prime}$, there exists a set $S \subseteq V$ such that $S^{\prime}=(V-S) \cup\left[\cup_{v \in S}\left\{v^{1}, v^{2}, \ldots, v^{n+1}\right\}\right]$.

Claim 3.6. Let $S$ be a maximal IS of $G$. Then $S^{\prime}=(V-S) \cup\left[\cup_{v \in S}\left\{v^{1}, v^{2}, \ldots, v^{n+1}\right\}\right]$ is a minimal $\mathrm{VC}$ of $G^{\prime}$.

Claim 3.7. Let $S^{\prime}$ be a minimal $\mathrm{VC}$ in $G^{\prime}$. If $V-S^{\prime}$ is not a maximal IS of $G$, then there exists a minimal VC $S^{\prime \prime}$ of $G^{\prime}$ such that $V-S^{\prime \prime}$ is a maximal IS of $G$ and, moreover,

(a) $\left|S^{\prime \prime}\right|>\left|S^{\prime}\right|$,

(b) $\left|V-S^{\prime \prime}\right|>\left|V-S^{\prime}\right|$ and

(c) $\left|S^{\prime \prime}\right|=n\left(\left|V-S^{\prime \prime}\right|+1\right)$.

Proof. Note that, for any VC $S^{\prime}$ of $G^{\prime}, V \cap S^{\prime}$ is a VC of $G$. Hence, $V-S^{\prime}=$ $V-\left(V \cap S^{\prime}\right)$ is an independent set of $G$. Let $S^{\prime}$ be a minimal VC of $G^{\prime}$ for which $V-S^{\prime}$ is not a maximal independent set of $G$. Then we can always extend $\left(V-S^{\prime}\right)$ to a unique maximal IS $S$ of $G$ (in polynomial time) by introducing vertices of $G$ one by one in the order $v_{1}, v_{2}, \ldots, v_{n}$ while maintaining the independence property. Hence, $S \supset\left(V-S^{\prime}\right)$. By Claim 3.6, $S^{\prime \prime}=(V-S) \cup\left[\cup_{v \in S}\left\{v^{1}, v^{2}, \ldots, v^{n+1}\right\}\right]$ is a minimal $\mathrm{VC}$ of $G^{\prime}$ and $\left|S^{\prime \prime}\right|=n(|S|+1)$. Now we show that $S=V-S^{\prime \prime}$. For this first note that $S \subseteq V$ as $S$ is a maximal independent set of $G$. Next, let $u \in S$, then from the definition of $S^{\prime \prime}$ it follows that $u \notin S^{\prime \prime}$, so $u \in V-S^{\prime \prime}$. Hence, $S \subseteq V-S^{\prime \prime}$. Also, if $u \in V-S^{\prime \prime}$, then $u \notin S^{\prime \prime}$, i.e. $u \notin V-S$, so $u \in S$. Hence, $S \supseteq V-S^{\prime \prime}$. Thus $S=V-S^{\prime \prime}$. From this it follows that $V-S^{\prime \prime}$ is a maximal independent set of $G$. 
From Claim 3.5, we have $\left|S^{\prime}\right|=\left|V \cap S^{\prime}\right|+(n+1)\left|V-\left(V \cap S^{\prime}\right)\right|=n(n+1)-$ $n\left|V \cap S^{\prime}\right|=n+n\left|V-S^{\prime}\right|=n\left(\left|V-S^{\prime}\right|+1\right)$. Since $|S|>\left|V-S^{\prime \prime}\right|$, it follows that $\left|S^{\prime \prime}\right|>\left|S^{\prime}\right|$. Also (b) and (c) follow from the fact that $S=V-S^{\prime \prime}$.

Claim 3.8. $S \subseteq V$ is a maximum IS of $G$ iff $S^{\prime}=(V-S) \cup\left[\cup_{v \in S}\left\{v^{1}, v^{2}, \ldots, v^{n+1}\right\}\right]$ is a maximum cardinality minimal $\mathrm{VC}$ of $G^{\prime}$.

Proof. Let $S$ be a maximum IS of $G$. By Claim 3.6, $S^{\prime}$ is a minimal VC of $G^{\prime}$. If $S^{\prime}$ is not a maximum cardinality minimal $\mathrm{VC}$ of $G^{\prime}$, then using Claim 3.7 there exists a minimal VC $S^{\prime \prime}$ of $G^{\prime}$ such that $\left|S^{\prime \prime}\right|>\left|S^{\prime}\right|, S=V-S^{\prime \prime}$ is a maximal IS of $G$ and $\left|S^{\prime \prime}\right|=n(|S|+1)$. As $\left|S^{\prime}\right|<\left|S^{\prime \prime}\right|,\left|S^{\prime}\right|=n(|S|+1)$ and $\left|S^{\prime \prime}\right|=n(|\tilde{S}|+1)$, it follows that $|S|<|\tilde{S}|$, which is a contradiction. Hence $S^{\prime}$ is a maximum cardinality minimal VC in $G$.

Let $S^{\prime}$ be a maximum cardinality minimal $\mathrm{VC}$ of $G^{\prime}$. Then by Claim 3.7, $S=V-S^{\prime}$ is a maximal IS of $G$ and $\left|S^{\prime}\right|=n(|S|+1)$. We claim that $S$ is a maximum IS in $G$. Suppose there exists a maximal IS $S^{*} \subseteq V$ of $G$ with $\left|S^{*}\right|>|S|$. By Claim 3.6, $\hat{S}=\left(V-S^{*}\right) \cup\left[\cup_{v \in S^{*}}\left\{v^{1}, v^{2}, \ldots, v^{n+1}\right\}\right]$ is a minimal VC in $G^{\prime}$ and $|\hat{S}|=n\left(\left|S^{*}\right|+1\right)$. Since $\left|S^{*}\right|>|S|$, it follows that $|\hat{S}|>\left|S^{\prime}\right|$, which is a contradiction. Hence, $S$ is a maximum IS of $G$.

We now complete the proof Theorem 3.4. Let $\alpha(G)$ denote the independence number and $\beta(G)$ denote the size of a maximum cardinality minimal VC in $G$. Hence, from Claim 3.8, we have $\beta\left(G^{\prime}\right)=n(\alpha(G)+1)$. Now let $S^{\prime}$ be any minimal VC of $G^{\prime}$. If $V-S^{\prime}$ is a maximal IS of $G$, then to $S^{\prime}$ we associate $S=V-S^{\prime}$ as the feasible solution of MAX-IS for $G$. If $V-S^{\prime}$ is not a maximal IS of $G$, then let $S^{\prime \prime}$ be the minimal VC of $G^{\prime}$ corresponding to $S^{\prime}$ as in Claim 3.7, so that $S=V-S^{\prime \prime}$ is a maximal IS of $G$ and $\left|S^{\prime}\right|<\left|S^{\prime \prime}\right|=n(|S|+1)$. To this minimal VC $S^{\prime}$ of $G^{\prime}$ we associate $S$ as the feasible solution of MAX-IS for $G$. Hence for any minimal VC $S^{\prime}$ of $G^{\prime}$ we have

$$
\begin{aligned}
\frac{\alpha(G)}{|S|} & =\frac{n \alpha(G)}{n|S|}=\frac{\beta\left(G^{\prime}\right)-n}{\left|S^{\prime \prime}\right|-n}=\frac{\beta\left(G^{\prime}\right)}{\left|S^{\prime \prime}\right|-n}-\frac{n}{\left|S^{\prime \prime}\right|-n} \\
& =\frac{\beta\left(G^{\prime}\right)}{\left|S^{\prime \prime}\right|} \cdot \frac{\left|S^{\prime \prime}\right|}{\left|S^{\prime \prime}\right|-n}-\frac{1}{|S|}=\frac{\beta\left(G^{\prime}\right)}{\left|S^{\prime \prime}\right|} \cdot \frac{n(|S|+n)}{n|S|}-\frac{1}{|S|} \\
& =\frac{\beta\left(G^{\prime}\right)}{\left|S^{\prime \prime}\right|}+\frac{1}{|S|}\left(\frac{\beta\left(G^{\prime}\right)}{\left|S^{\prime \prime}\right|}-1\right) \\
& \leq \frac{\beta\left(G^{\prime}\right)}{\left|S^{\prime \prime}\right|}+\frac{\beta\left(G^{\prime}\right)}{\left|S^{\prime \prime}\right|}-1 \quad\left(\text { since } \frac{\beta\left(G^{\prime}\right)}{\left|S^{\prime \prime}\right|} \geq 1 \text { and }|S| \geq 1\right) \\
& <2 \frac{\beta\left(G^{\prime}\right)}{\left|S^{\prime \prime}\right|} \leq 2 \frac{\beta\left(G^{\prime}\right)}{\left|S^{\prime}\right|} .
\end{aligned}
$$

Let $N$ be the number of vertices in $G^{\prime}$. Since $N=n^{2}+2 n$ and $N \leq 2 n^{2}$, for $n \geq 2$. Now, for any $\epsilon>0, n^{1-\epsilon} \geq \frac{N^{\frac{1}{2}(1-\epsilon)}}{2^{\frac{1}{2}(1-\epsilon)}} \geq \frac{N^{\frac{1}{2}(1-\epsilon)}}{\sqrt{2}} \cdot 2^{\frac{\epsilon}{2}} \geq \frac{1}{\sqrt{2}} N^{\frac{1}{2}(1-\epsilon)}$. From Theorem 2.4, it now follows that, unless NP $=\mathrm{ZPP}$, for any $\epsilon>0$, there exists no 
polynomial time algorithm to approximate $\beta\left(G^{\prime}\right)$ within a factor of $\frac{1}{2 \sqrt{2}} N^{\frac{1}{2}(1-\epsilon)}$, where $N$ is the number of vertices in $G^{\prime}$. Hence the theorem follows.

We also have:

Theorem 3.9. Unless $P=\mathrm{NP}$, for any $\epsilon>0$, there is no polynomial time algorithm to approximate $M A X-M I N-V C$ within a factor of $\frac{1}{2 \sqrt[4]{2}} n^{\frac{1}{4}-\epsilon}$, where $n$ is the number of vertices in an instance.

Proof. Same as that of Theorem 3.4, and the fact that $\frac{1}{\sqrt[4]{2}} N^{\frac{1}{4}(1-2 \epsilon)} \leq n^{\frac{1}{2}-\epsilon}$.

Regarding MAX-MIN-FVS, we have similar results. First we shall consider this problem for digraphs.

Theorem 3.10. Unless $\mathrm{NP}=\mathrm{ZPP}$, for any $\epsilon>0$, there exists no polynomial time algorithm to approximate $M A X-M I N-F V S$, for general digraphs, within a factor of $\frac{1}{4} n^{\frac{1}{2}-\epsilon}$, where $n$ is the number of vertices in an instance.

Proof. We prove this by an $S$-reduction from MAX-MIN-VC to MAX-MIN-FVS as follows.

Let $G=(V, E)$ be a graph (an instance of MAX-MIN-VC). Construct an instance $G^{\prime}=\left(V^{\prime}, A^{\prime}\right)$ of MAX-MIN-FVS from $G$ with $V^{\prime}=\cup_{v_{i} \in V}\left\{v_{i}^{1}, v_{i}^{2}\right\}$ and $A^{\prime}=\left[\cup_{v_{i} \in V}\left\{\left(v_{i}^{1}, v_{i}^{2}\right)\right\}\right] \cup\left[\cup_{\left\{v_{i}, v_{j}\right\} \in E}\left\{\left(v_{i}^{2}, v_{j}^{1}\right),\left(v_{j}^{2}, v_{i}^{1}\right)\right\}\right]$. In other words, for each $v_{i} \in V, G^{\prime}$ has 2 vertices $v_{i}^{1}, v_{i}^{2}$ and an $\operatorname{arc}\left(v_{i}^{1}, v_{i}^{2}\right)$. Also for each $\left\{v_{i}, v_{j}\right\} \in E G^{\prime}$ has $\left(v_{i}^{2}, v_{j}^{1}\right)$ and $\left(v_{j}^{2}, v_{i}^{1}\right)$. Hence, $G^{\prime}$ has $2 n$ vertices and $n+2 m$ arcs. See Figure 3 for an example.

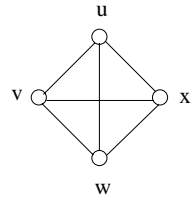

$\mathrm{G}=(\mathrm{V}, \mathrm{E})$

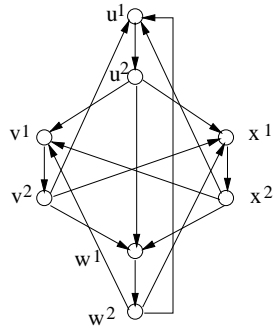

$\mathrm{G}^{\prime}=\left(\mathrm{V}^{\prime}, \mathrm{E}^{\prime}\right)$

Figure 3. An instance $G$ of MAX-MIN-VC and the corresponding instance $G^{\prime}$ of MAX-MIN-FVS.

First we establish two claims.

Claim 3.11. For any $C \subseteq V$,

(1) $C$ is a VC of $G$ iff $F=\left\{v_{i}^{1} \mid v_{i} \in C\right\}$ is an FVS of $G^{\prime}$.

(2) $C$ is a minimal VC of $G$ iff $F$ is a minimal FVS of $G^{\prime}$. 
Proof. (1) Let $C$ be a VC of $G$. We will show that $F$ is a FVS of $G^{\prime}$. Every dicycle in $G^{\prime}$ contains the sequence of four vertices $\left(v_{i}^{1}, v_{i}^{2}, v_{j}^{1}, v_{j}^{2}\right)$ for some $\left\{v_{i}, v_{j}\right\} \in E$. Since $C$ is a VC, it follows that at least one of $v_{i}^{1}$ and $v_{j}^{1}$ is in $F$. Hence, $F$ is an FVS of $G^{\prime}$.

Conversely, let $F$ be an FVS of $G$. Suppose $C$ is not a VC of $G$. Then there exists an edge $\left\{v_{i}, v_{j}\right\} \in E$ none of $v_{i}$ and $v_{j}$ is in $C$. Hence, the dicycle $\left(v_{i}^{1}, v_{i}^{2}, v_{j}^{1}, v_{j}^{2}, v_{i}^{1}\right)$ is in $G^{\prime}-F$, and hence, $F$ is not an FVS of $G^{\prime}$ which is a contradiction.

(2) Let $C$ be a minimal VC of $G$. Thus, for any $v_{i} \in C$, there exists a $v_{j} \in V-C$ such that $\left\{v_{i}, v_{j}\right\} \in E$. Hence for any $v_{i}^{1} \in F$, there is some $v_{j} \in V-C$ such that the dicycle $\left(v_{i}^{1}, v_{i}^{2}, v_{j}^{1}, v_{j}^{2}, v_{i}^{1}\right)$ contains one and only one vertex namely $v_{i}^{1}$ in $F$. Hence $F$ is a minimal FVS of $G^{\prime}$.

Conversely, let $F$ be a minimal FVS of $G^{\prime}$ and suppose $C$ is not a minimal VC of $G$. Let $C^{\prime} \subset C$ be a minimal VC of $G$. By (1), $F^{\prime}$ is an FVS of $G^{\prime}$ and $F^{\prime} \subset F$, which is a contradiction. So $C$ must be a minimal VC of $G$.

Claim 3.12. Let $F$ be any minimal FVS of $G^{\prime}$. Then:

(1) for any $v_{i} \in V, F \cap\left\{v_{i}^{1}, v_{i}^{2}\right\}$ is either empty or singleton;

(2) for any $v_{i} \in V$ such that $F \cap\left\{v_{i}^{1}, v_{i}^{2}\right\} \neq \phi, F^{\prime}=F-\left\{v_{i}^{1}, v_{i}^{2}\right\}+v_{i}^{1}$ is also a minimal FVS of $G^{\prime}$

(3) there is a minimal FVS $F^{\prime}$ of $G^{\prime}$ such that $\left|F^{\prime}\right|=|F|$ and $F^{\prime}=\left\{v_{i}^{1} \mid v_{i} \in C\right\}$ for some minimal VC $C$ of $G$ such that $|C|=\left|F^{\prime}\right|$.

Proof. (1) This follows because $F$ is a minimal FVS and every dicycle of $G^{\prime}$ contains either both $v_{i}^{1}$ and $v_{i}^{2}$ or none at all.

(2) If $F$ contains $v_{i}^{1}$ then $F=F^{\prime}$. If $F$ contains $v_{i}^{2}$, then $F^{\prime}=F-v_{i}^{2}+v_{i}^{1}$ and, as every cycle in $G^{\prime}$ containing $v_{i}^{2}$ must contain $v_{i}^{1}$ and vice versa, $F^{\prime}$ must be a minimal FVS of $G^{\prime}$.

(3) By repeated application of (2), we get a minimal FVS $F^{\prime}$ of $G^{\prime}$ such that $\left|F^{\prime}\right|=|F|$ and any vertex $v \in F^{\prime}$ is $v_{i}^{1}$ for some $v_{i} \in V$. Let $C=\left\{v_{i} \mid v_{i}^{1} \in F^{\prime}\right\}$. Then $F^{\prime}=\left\{v_{i}^{1} \mid v_{i} \in C\right\}$. Now by Claim 3.11, as $F^{\prime}$ is a minimal FVS of $G^{\prime}, C$ is a minimal $\mathrm{VC}$ of $G$.

Now coming back to the proof of Theorem 3.10, let $F_{o}$ be a maximum minimal FVS of $G^{\prime}$ and $F$ be any minimal FVS of $G^{\prime}$. By Claim 3.12, without loss of generality we can assume that every vertex in $F_{o}$ (respectively, in $F$ ) is of the form $v_{i}^{1}$ for some $v_{i} \in V$. Also by Claim 3.12, $C_{o}=\left\{v_{i} \mid v_{i}^{1} \in F_{o}\right\}$, (respectively, $C=\left\{v_{i} \mid v_{i}^{1} \in F\right\}$ ) is a maximum minimal $\mathrm{VC}$ (respectively, minimal $\mathrm{VC}$ ) of $G$, and $\left|C_{o}\right|=\left|F_{o}\right|$ (respectively, $\left.|C|=|F|\right)$. Hence $\frac{\left|C_{o}\right|}{|C|}=\frac{\left|F_{o}\right|}{|F|}$.

Let $N=\left|V^{\prime}\right|$. Then $N=2 n$. Now for any $\epsilon>0, \frac{1}{2 \sqrt{2}} n^{\frac{1}{2}-\epsilon}=\frac{1}{2 \sqrt{2}} \frac{(2 n)^{\frac{1}{2}-\epsilon}}{2^{\frac{1}{2}-\epsilon}}=$ $\frac{1}{4} N^{\frac{1}{2}-\epsilon} \cdot 2^{\epsilon} \geq \frac{1}{4} N^{\frac{1}{2}-\epsilon}$. Hence, by Theorem 3.4, the result follows. 
We also have:

Theorem 3.13. Unless $P=\mathrm{NP}$, for any $\epsilon>0$, there exists no polynomial time algorithm to approximate $M A X-M I N-F V S$, for general digraphs, within a factor of $\frac{1}{2 \sqrt{2}} n^{\frac{1}{4}-\epsilon}$, where $n$ is the number of vertices in an instance.

Towards MAX-MIN-FVS for general graphs we have:

Theorem 3.14. Unless $\mathrm{NP}=\mathrm{ZPP}$, for any $\epsilon>0$, there exists no polynomial time algorithm to approximate MAX-MIN-FVS, for general graphs, within a factor of $\frac{1}{4} n^{\frac{1}{2}-\epsilon}$, where $n$ is the number of vertices in an instance.

Proof. We shall prove this by establishing an $S$-reduction from MAX-MIN-VC. For any given instance of MAX-MIN-VC, i.e. a graph $G=(V, E)$, construct an instance $G^{\prime}=\left(V^{\prime}, E^{\prime}\right)$ from $G$ by introducing a new vertex $t$ and connecting each vertex of $G$ to $t$ by an edge. Hence in $G^{\prime}, V^{\prime}=V \cup\{t\}$ and $E^{\prime}=E \cup\left\{\left(v_{i}, t\right) \mid v_{i} \in V\right\}$. Clearly, $\left|V^{\prime}\right|=n+1$.

Obviously, if $C \subseteq V$ is a minimal VC of $G$ then $C$ is also a minimal FVS of $G^{\prime}$. It can be easily observed that, for any graph $G, G^{\prime}$ has a maximum cardinality minimal FVS without containing the vertex $t$. Also, if $F \subseteq V^{\prime}$ is a minimal FVS of $G^{\prime}$ with $t \notin F$, then $F$ is a minimal VC of $G$. From this, it follows that, if $C_{o}$ is a maximum cardinality minimal $\mathrm{VC}$ of $G$ then $C_{o}$ is also a maximum cardinality minimal FVS of $G^{\prime}$. It is important to observe that if $F$ is a minimal FVS of $G^{\prime}$ with $t \in F$ then in polynomial time one can construct an FVS $F^{\prime}$ of $G^{\prime}$ with $|F| \leq\left|F^{\prime}\right|$ and $t \notin F^{\prime}$. Since an optimal solution of MAX-MIN-FVS for the instance $G^{\prime}$ does not contain the special vertex $t$, without loss of generality, we can assume that a minimal FVS of $G^{\prime}$ does not contain the vertex $t$. Hence, this is an $S$-reduction with the parameter $c=1$.

Now, let $N=\left|V^{\prime}\right|$. Hence $N=n+1$. As $N \geq 2$, for any $\epsilon>0$, we have $(N-1)^{\frac{1}{2}-\epsilon} \geq \frac{1}{\sqrt{2}} N^{\frac{1}{2}-\epsilon}$. Thus $\frac{1}{2 \sqrt{2}} n^{\frac{1}{2}-\epsilon} \geq \frac{1}{4} N^{\frac{1}{2}-\epsilon}$. Hence, by Theorem 3.4, the result follows.

Similarly we can prove:

Theorem 3.15. Unless $P=\mathrm{NP}$, for any $\epsilon>0$, there exists no polynomial time algorithm to approximate MAX-MIN-FVS, for general graphs, within a factor of $\frac{1}{2 \sqrt{2}} n^{\frac{1}{4}-\epsilon}$, where $n$ is the number of vertices in an instance.

\section{HARDNESS RESULTS FOR BOUNDED DEGREE GRAPHS}

In this section we establish APX-hardness of MIN-FVS and MAX-MIN-FVS for certain restricted class of undirected graphs. Regarding MIN-FVS, it is known that it can be solved in polynomial time for all graphs of maximum degree 3 [33], but it is not known whether MIN-FVS is NP-complete for graphs of maximum degree 4 or 5 . However, as suggested by Fujito [15], it is easy to show that:

Proposition 4.1. MIN-W-FVS- $\leq 4$ is NP-complete and also APX-complete. 
Proof. From a 3-regular graph (an instance of MIN-VC-3) construct a vertex weighted graph $\left(G^{\prime}=\left(V^{\prime}, E^{\prime}\right), w\right)$ of degree at most 4 as follows. $V^{\prime}=V \cup\left\{v^{i} \mid 1 \leq\right.$ $\left.i \leq \frac{n}{2}\right\}, E^{\prime}=E \cup\left[\cup_{1 \leq i \leq \frac{n}{2}}\left\{\left\{v_{2 i-1}, v^{i}\right\},\left\{v_{2 i}, v^{i}\right\}\right\}\right] \cup\left[\left\{\left\{v^{i}, v^{i+1}\right\} \mid 1 \leq i<\frac{n}{2}\right\}\right]$, and $w(v)=1$ for $v \in V$ and $w(v)=M$ for $v \in V^{\prime}-V$, where $M>n$ is a positive large integer.

It can be shown that, any FVS $F$ of $G^{\prime}$ with $w(F)<n$ is also a VC of $G$ and any VC $C$ of $G$ is also an FVS of $G^{\prime}$. From this, NP-completeness of MIN-W-FVS- $\leq 4$ follows. Further, as MIN-W-FVS $\in$ APX [4], MIN-W-FVS- $\leq 4 \in$ APX. Now, if $C_{o}$ is a minimum VC of $G$ then $C_{o}$ is also a minimum FVS of $G^{\prime}$ and they are of same cost. To an FVS $F$ of $G^{\prime}$ with $w(F)=\sum_{v \in F} w(v) \geq n$ we assign $V$ as a vertex cover of $G$. Hence, from any FVS $F$ of $G^{\prime}$ we can construct a VC $C$ of $G$ such that $|C| \leq w(F)$. So $\frac{|C|}{\left|C_{o}\right|} \leq \frac{w(F)}{w\left(F_{o}\right)}$. From this it follows that, MIN-VC-3 $\leq_{A P}$ MIN-W-FVS- $\leq 4$ with $\alpha=1$, and so, MIN-W-FVS- $\leq 4$ is APX-complete as MIN-VC-3 is so [1].

Next we show that MIN-FVS-6 is APX-complete.

Theorem 4.2. MIN-FVS-6 is APX-complete.

Proof. As MIN-FVS is in class APX [4], it is enough to show that MIN-FVS-6 is APX-hard. Towards this we will show that MIN-VC-3 $\leq_{L}$ MIN-FVS-6.

Let $G=(V, E)$ be a 3-regular graph. From $G$ construct a 6-regular graph $G^{\prime}=$ $\left(V^{\prime}, E^{\prime}\right)$ as follows: for every edge $\left\{v_{i}, v_{j}\right\} \in E$, let $V_{i j}=\left\{v_{i j}^{1}, v_{i j}^{2}, v_{i j}^{3}, v_{i j}^{4}, v_{i j}^{5}, v_{i j}^{6}, v_{i j}^{7}\right\}$ be the set of seven new vertices and $H_{i j}=\left(V_{i j}, E_{i j}\right)$ be the graph obtained from the complete graph on $V_{i j}$ by removing the edge $\left\{v_{i j}^{1}, v_{i j}^{7}\right\}$. Now $V^{\prime}=V \cup$ $\left[\cup_{\left\{v_{i}, v_{j}\right\} \in E} V_{i j}\right]$ and $E^{\prime}=E \cup\left[\cup_{\left\{v_{i}, v_{j}\right\} \in E}\left[E_{i j} \cup\left\{\left\{v_{i}, v_{i j}^{1}\right\},\left\{v_{i j}^{7}, v_{j}\right\}\right\}\right]\right]$, see Figure 4. Clearly $G^{\prime}$ is 6-regular.
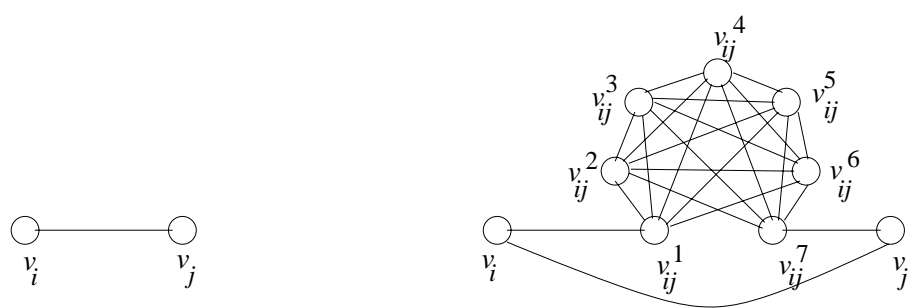

FiguRE 4. An edge $\left\{v_{i}, v_{j}\right\} \in E$ and corresponding subgraph in $G^{\prime}$.

Next we establish a few claims. But first note that if $F$ is an FVS of $G^{\prime}$, then $F$ contains at least 4 vertices from $V_{i j}$.

Claim 4.3. Let $F$ be any FVS of $G^{\prime}$ containing exactly 4 vertices from $V_{i j}$ for some $\left\{v_{i}, v_{j}\right\} \in E$. Then $F$ must contain either $v_{i}$ or $v_{j}$.

Proof. If $v_{i j}^{1} \in F$, then then the three vertices $v_{i j}^{p}, v_{i j}^{q}, v_{i j}^{r}$ not in $F$ form a cycle in $G^{\prime}$. Hence, $F$ can not be an FVS of $G^{\prime}$. So $v_{i j}^{1} \notin F$. By similar argument, one can show that $v_{i j}^{7} \notin F$. Hence, $F$ contains 4 vertices from $v_{i j}^{2}, v_{i j}^{3}, v_{i j}^{4}, v_{i j}^{5}, v_{i j}^{6}$. 
Let $v_{i j}^{l} \notin F$ for some $2 \leq l \leq 6$. Since $F$ is an FVS of $G$ and the vertices $v_{i}, v_{i j}^{1}, v_{i j}^{l}, v_{i j}^{7}, v_{j}$ form a cycle in $G^{\prime}, F$ must contain either $v_{i}$ or $v_{j}$.

To an FVS $F$ of $G^{\prime}$, we associate the set $C$ of vertices in $G$ defined as $C=$ $(F \cap V) \cup\left\{v_{i}|| F \cap V_{i j} \mid \geq 5\right.$ and $\left.i<j\right\}$.

Claim 4.4. $C$ is a VC of $G$ and $|F| \geq|C|+4|E|=|C|+6 n$.

Proof. If $C$ is not a VC of $G$, then there exists $\left\{v_{i}, v_{j}\right\} \in E$ such that $C \cap\left\{v_{i}, v_{j}\right\}=$ $\phi$. By the definition of $C$, it follows that $\left|F \cap V_{i j}\right| \leq 4$ and $F \cap\left\{v_{i}, v_{j}\right\}=\phi$. If $\left|F \cap V_{i j}\right|<4$, then $F$ is not an FVS of $G^{\prime}$, so $\left|F \cap V_{i j}\right|=4$. By Claim $4.3, F$ must contain either $v_{i}$ or $v_{j}$. Otherwise $F$ can not be an FVS of $G^{\prime}$. This contradicts that $F \cap\left\{v_{i}, v_{j}\right\}=\phi$. Hence, $C$ is a $\mathrm{VC}$ of $G$.

Now $|F|=4|E|+|F \cap V|+\left|\left\{v_{i}|| F \cap V_{i j} \mid \geq 5, i<j\right\}\right|$, as $F$ contains at least 4 vertices from $V_{i j}$ for each $\left\{v_{i}, v_{j}\right\} \in E$, and for the edges $\left\{v_{i}, v_{j}\right\} \in E$ such that $\left|F \cap V_{i j}\right| \geq 5, F$ contains at least one more vertex from $V_{i j}$ in addition to 4 vertices already considered. Hence, $|F| \geq|C|+4|E|=|C|+6 n$ as $G$ is a 3-regular and $|E|=\frac{3}{2} n$.

Claim 4.5. For any $\mathrm{VC} C$ in $G$, the set $F=C \cup\left\{v_{i j}^{2}, v_{i j}^{3}, v_{i j}^{4}, v_{i j}^{5} \mid\left\{v_{i}, v_{j}\right\} \in E\right\}$ is an FVS of $G^{\prime}$ such that $C=F \cap V$ and $|F|=|C|+6 n$.

Proof. Take any cycle $K$ in $G^{\prime}$. If $K$ is a cycle in $G$, then $K$ has a vertex in $C \subset F$. Hence, $F$ breaks all cycles in $G$. If $K$ contains a vertex in $G^{\prime}$ and if $K$ contains none of $v_{i j}^{2}, v_{i j}^{3}, v_{i j}^{4}, v_{i j}^{5}$ for any $\left\{v_{i}, v_{j}\right\} \in E$, then it must contain both $v_{i}$ and $v_{j}$, and so $K$ has a common vertex with $C \subset F$. Thus every cycle $K$ in $G^{\prime}$ intersects $F$. So $F$ is an FVS of $G^{\prime}$.

Clearly, $C=F \cap V$ and so $|F|=|C|+6 n$.

Claim 4.6. If $F_{o}$ is a minimum FVS of $G^{\prime}$, then the associated set $C_{o}$ is a minimum VC of $G$ and $\left|F_{o}\right|=\left|C_{o}\right|+n$.

Proof. Let $C_{o}$ be the $\mathrm{VC}$ of $G$ associated with $F_{o}$, and $F$ be the FVS of $G^{\prime}$ associated with $C_{o}$ as constructed in proof of Claim 4.5. Then $\left|F_{o}\right| \geq\left|C_{o}\right|+6 n=$ $|F|$. But as $F_{o}$ is a minimum FVS of $G^{\prime}$, equality holds, i.e., $\left|F_{o}\right|=\left|C_{o}\right|+n$. From this it follows that $C_{o}$ is a minimum VC of $G$.

Now to complete the proof of Theorem 4.2, first note that, any $\mathrm{VC} C$ in a 3-regular graph $G$ contains at least $\frac{n}{2}$ vertices. To see this, if possible let $|C|<\frac{n}{2}$. Then note that $V-C$ is an IS of $G$ and the number of edges between $V-C$ and $C$ is $3|V-C|=3 n-3|C|>\frac{3}{2} n$, the number of edges in $G$, which is not possible.

Now note that $\left|F_{o}\right|=\left|C_{o}\right|+6 n \leq\left|C_{o}\right|+12\left|C_{o}\right|=13\left|C_{o}\right|$. Hence, the first inequality of $L$-reduction holds with $\alpha=13$. Next, for any FVS $F$ of $G^{\prime},|F|-$ $\left|F_{o}\right| \geq|C|+6 n-\left|C_{o}\right|-6 n=|C|-\left|C_{o}\right|$. So the second inequality of $L$-reduction holds with $\beta=1$. 
Next we shall consider MAX-MIN-FVS. Before that we note the following:

Lemma 4.7. For any FVS $F$ of a 6-regular graph $G=(V, E),|F|>\frac{2}{5} n$.

Proof. Observe that, $G$ has $3 n$ edges and the subgraph $G[V-F]$ induced by $V-F$, is a forest. Since $G[V-F]$ has at most $n-|F|-1$ edges and the number of edges removed by removing $F$ is at most $6|F|$, we have $3 n-6|F| \leq n-|F|-1$, i.e. $|F|>\frac{2}{5} n$.

Theorem 4.8. $M A X-M I N-F V S-\leq 9$ is $A P X$-hard.

Proof. Let $G=(V, E)$ be a 6-regular graph. Construct a graph $G^{\prime}=\left(V^{\prime}, E^{\prime}\right)$ of degree at most 9 as follows: $V^{\prime}=V \cup\left\{v^{1}, v^{2}, v^{3} \mid v \in V\right\}$ and $E^{\prime}=E \cup$ $\left\{\left(v, v^{1}\right),\left(v, v^{2}\right),\left(v, v^{3}\right),\left(v^{1}, v^{2}\right),\left(v^{1}, v^{3}\right) \mid v \in V\right\}$ (see Fig. 5).

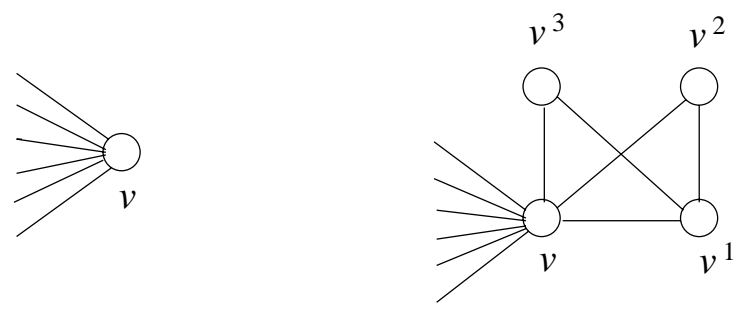

Figure 5. A vertex $v$ in $G$ and its corresponding neighbors in $G^{\prime}$.

Let $F$ be any minimal FVS of $G^{\prime}$. Note that, for any $v \in V-F, F$ contains either $v^{1}$ or both $v^{2}$ and $v^{3}$. Further, if $v \in F \cap V$, then $F \cap\left\{v^{1}, v^{2}, v^{3}\right\}=\phi$. To $F$ we associate $C=F \cap V$, which is clearly an FVS of $G$. Note that $|F| \leq$ $|C|+2|V-F|=|C|+2|V-C|=2 n-|C|$.

If $C$ is an FVS of $G$, then we show that $F=C \cup\left\{v^{2}, v^{3} \mid v \in V-C\right\}$ is a minimal FVS of $G^{\prime}$ and $|F|=2 n-|C|$. Clearly $F$ is an FVS of $G^{\prime}$. $F$ is a minimal FVS of $G^{\prime}$ as (a) any $v \in C$ can not be dropped from $F$ as $\left\{v^{1}, v^{2}, v^{3}\right\} \cap F=\phi$ and $G^{\prime}\left[\left\{v, v^{1}, v^{2}, v^{3}\right\}\right]$ contains cycles; and (b) for any $v \in V-C$, none of $v^{2}$ and $v^{3}$ can be dropped from $F$ as $G^{\prime}\left[\left\{v, v^{1}, v^{2}\right\}\right]$ and $G^{\prime}\left[\left\{v, v^{1}, v^{3}\right\}\right]$ contain cycles. Obviously $|F|=2 n-|C|$.

Let $F_{o}$ be a maximum minimal FVS of $G^{\prime}$ and $C_{o}=F_{o} \cap V$. We claim that $\left|F_{o}\right|=2 n-\left|C_{o}\right|$. For, if $\left|F_{o}\right|<2 n-\left|C_{o}\right|$, then $F=C_{o} \cup\left\{v^{2}, v^{3} \mid v \in V-C_{o}\right\}$ is a minimal FVS of $G^{\prime}$ with $|F|=2 n-\left|C_{o}\right|>\left|F_{o}\right|$ contradicting the assumption that $F_{o}$ is a maximum minimal FVS of $G^{\prime}$. Next we claim that $C_{o}$ is a minimum FVS of $G$. If possible, let $C$ be an FVS of $G$ with $|C|<\left|C_{o}\right|$. Then $F=$ $C \cup\left\{v^{2}, v^{3} \mid v \in V-C\right\}$ is a minimal FVS of $G^{\prime}$ with $|F|=2 n-|C|$. But as $|C|<\left|C_{o}\right|,|F|=2 n-|C|>2 n-\left|C_{o}\right|=\left|F_{o}\right|$, which is a contradiction to our assumption that $F_{o}$ is a maximum minimal FVS of $G^{\prime}$.

Now, $\left|F_{o}\right|=2 n-\left|C_{o}\right|<5\left|C_{o}\right|-\left|C_{o}\right|=4\left|C_{o}\right|$, (by Lem. 4.7). So, the first inequality of $L$-reduction holds with $\alpha=4$. Next, for any minimal FVS $F$ of $G^{\prime},\left|F_{o}\right|-|F| \geq 2 n-\left|C_{o}\right|-2 n+|C|=|C|-\left|C_{o}\right|$. So the second inequality of $L$-reduction holds with $\beta=1$. 
Next we shall consider MAX-MIN-VC. First we have the following two simple lemmas:

Lemma 4.9. For any 3-regular graph $G=(V, E)$ and any maximal IS $I$ in $G$, $|I| \geq \frac{1}{4}|V|$.

Proof. Let $I$ be any maximal IS of $G$. Then the subgraph $G[V-I]$ of $G$ induced by $V-I$ is of degree at most 2. Thus $G[V-I]$ contains at most $|V-I|$ edges. Thus the number of edges in $G$ is bounded above by $3|I|+|V-I|$. Hence $\frac{3}{2}|V| \leq|V|+2|I|$, i.e., $\frac{|V|}{4} \leq|I|$.

Lemma 4.10. MAX-MIN-VC is k-approximable for graphs of maximum degree $k, k \geq 1$, and having no isolated vertex.

Proof. Let $G=(V, E)$ be any graph of maximum degree $k, k \geq 1$, and having no isolated vertex. We show that any minimal $\mathrm{VC} C$ of $G$ is a $k$-approximable solution for MAX-MIN-VC. A minimal VC of $G$ can be obtained by the simple algorithm:

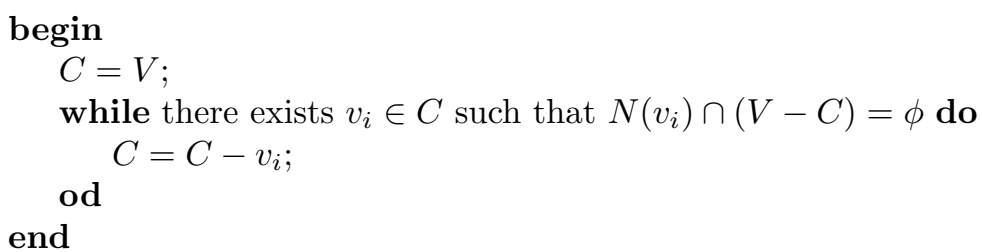

where $N\left(v_{i}\right)=\left\{v_{j} \mid\left\{v_{i}, v_{j}\right\} \in E\right\}$.

Claim 4.11. For any minimal VC $C$ of $G,|C| \leq \frac{k n}{k+1}$, where $n=|V|$.

Proof. As $C$ is a minimal VC of $G, V-C$ is a maximal IS of $G$. Also as $V-C$ is a maximal IS of $G$ and $G$ has no isolated vertices, for any $v_{i} \in V-C, v_{i}$ is not adjacent to any $w \in V-C$, but is adjacent to some $v_{j} \in C$. Let $S_{v_{i}}=\left\{v_{j} \in\right.$ $\left.C \mid\left\{v_{i}, v_{j}\right\} \in E\right\}$, for $v_{i} \in V-C$. Then $C=\cup_{v_{i} \in V-C} S_{v_{i}}$. Clearly, $\cup_{v_{i} \in V-C} S_{v_{i}} \subseteq C$. Conversely, let $v_{j} \in C$, then there exists $v_{i} \in V-C$ such that $\left\{v_{i}, v_{j}\right\} \in E$ and so $v_{j} \in S_{v_{i}}$. Hence $C \subseteq \cup_{v_{i} \in V-C} S_{v_{i}}$. Therefore, $|C| \leq \sum_{v_{i} \in V-C}\left|S_{v_{j}}\right| \leq k|V-C| \leq$ $n k-k|C|$. So $|C| \leq \frac{k n}{k+1}$.

Claim 4.12. For any $\operatorname{VC} C$ of $G,|C| \geq \frac{n}{k+1}$.

Proof. As $V-C$ is an IS of $G$ and $G$ has no isolated vertices, for any $v_{i} \in V-C$, $v_{i}$ is not adjacent to any $v_{k} \in V-C$ but must be adjacent to some $v_{j} \in C$. Let $S_{v_{j}}=\left\{v_{i} \in V-C \mid\left\{v_{i}, v_{j}\right\} \in E\right\}$, for $v_{j} \in C$. Then $V-C=\cup_{v_{j} \in C} S_{v_{j}}$. Hence, $k|C| \geq|V-C|$, i.e. $|C| \geq \frac{n}{k+1}$.

Let $C_{o}$ be a maximum cardinality minimal VC of $G$. By Claim 4.11, $\left|C_{o}\right| \leq \frac{k n}{k+1}$, and so by Claim $4.12, \frac{\left|C_{o}\right|}{|C|} \leq k$.

Now we have:

Theorem 4.13. MAX-MIN-VC- $\leq 5$ is APX-complete. 
Proof. Since MAX-MIN-VC is in class APX for bounded degree graphs (Lem. 4.10) and MAX-IS-3 is APX-complete [1], it is enough to show that MAX-IS-3 $\leq_{L}$ MAX$\mathrm{MIN}-\mathrm{VC}-\leq 5$.

Let $G=(V, E)$ be a 3-regular graph. From $G$ construct $G^{\prime}=\left(V^{\prime}, E^{\prime}\right)$ of degree at most 5 as follows: $V^{\prime}=V \cup\left[\cup_{v \in V}\left\{v^{1}, v^{2}\right\}\right]$ and $E^{\prime}=E \cup\left[\cup_{v \in V}\left\{\left\{v, v^{1}\right\},\left\{v, v^{2}\right\}\right\}\right]$ (see Fig. 6 for an example).

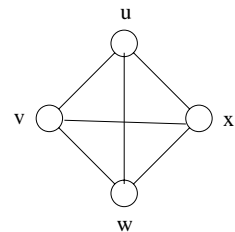

$G$

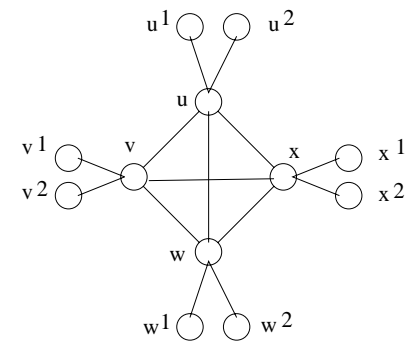

$G^{\prime}$

Figure 6

As in the proof of Theorem 3.4, we can prove the following:

(1) Any $\mathrm{VC} C$ of $G^{\prime}$ is a minimal $\mathrm{VC}$ of $G^{\prime}$ iff

(a) $v \in C \cap V \Rightarrow v^{1} v^{2} \notin C$ and

(b) $v \in(V-C) \Rightarrow\left\{v^{1}, v^{2}\right\} \subseteq C$.

Moreover, any minimal VC $C$ of $G^{\prime}$ is of the form $C=(V-I) \cup\left[\cup_{v \in I}\left\{v^{1}, v^{2}\right\}\right]$, for some IS $I$ of $G$ where $I=V-(C \cap V)$. The IS $I$ of $G$ defined above is the IS of $G$ associated with the minimal VC $C$ of $G^{\prime}$.

(2) $C$ is a minimal VC of $G^{\prime}$ iff the associated $I$ is a maximal IS of $G$, with $|C|=|I|+n$.

(3) $C_{o}$ is a maximum cardinality minimal $\mathrm{VC}$ of $G^{\prime}$ iff the associated $I_{O}$ is a maximum IS of $G$, with $\left|C_{o}\right|=\left|I_{o}\right|+n$.

Now, $\left|C_{o}\right|=\left|I_{o}\right|+n \leq\left|I_{o}\right|+4\left|I_{o}\right|=5\left|I_{o}\right|$ (by Lem. 4.9), so that, the first inequality of $L$-reduction holds with $\alpha=5$. Next, for any minimal $\operatorname{VC} C$ of $G^{\prime}$, $\left|C_{o}\right|-|C|=\left|I_{o}\right|+n-|I|-n=\left|I_{o}\right|-|I|$, so that, the second inequality of $L$-reduction holds with $\beta=1$.

\section{HARDNESS RESUltS FOR BOUNDED DEGREE DIGRAPHS}

We know that MIN-FAS is APX-hard [24] and MAX-SUBDAG is APX-complete [28] for general digraphs. In this section, we show that these problems remain APX-hard even for $k$-total-regular digraphs for all $k \geq 4$. We also show that MIN-MAX-SUBDAG is APX-hard for digraphs of maximum total degree 12 and MAX-MIN-VC is APX-hard for graphs of maximum degree 5. Regarding MINFAS, we first prove the following: 
Lemma 5.1. MIN-FAS- $k \leq_{L} M I N-F A S-(k+1)$, for all $k \geq 1$.

Proof. We construct in polynomial time, from a $k$-total-regular digraph $G=$ $(V, A)$, a $(k+1)$-total-regular digraph $G^{\prime}=\left(V^{\prime}, A^{\prime}\right)$ where $V^{\prime}=\left\{v^{1}, v^{2} \mid v \in V\right\}$ and $A^{\prime}=\left\{\left(u^{1}, v^{1}\right),\left(u^{2}, v^{2}\right) \mid(u, v) \in A\right\} \cup\left\{\left(v^{1}, v^{2}\right) \mid v \in V\right\}$. We shall denote $V^{i}=\left\{v^{i} \mid v \in V\right\}$ and $A^{i}=\left\{\left(u^{i}, v^{i}\right) \mid(u, v) \in A\right\}$, for $i=1,2$. From a minimal FAS $S^{\prime}$ of $G^{\prime}$ construct a minimal FAS $S$ of $G$ as follows: $S=\left\{(u, v) \mid\left(u^{1}, v^{1}\right) \in S^{1}\right\}$ where without loss of generality we assume that $S^{\prime}=S^{1} \cup S^{2}$ with $S^{1}$ and $S^{2}$ are minimal FASs of $G^{1}=\left(V^{1}, A^{1}\right)$ and $G^{2}=\left(V^{2}, A^{2}\right)$ respectively, and $\left|S^{1}\right| \leq\left|S^{2}\right|$. It is easy to see that, if $S_{o}^{\prime}$ is a minimum FAS of $G^{\prime}$, then the corresponding $S_{o}$ is a minimum FAS of $G$ and $\left|S_{o}^{\prime}\right|=2\left|S_{o}\right|$. Further, for any minimal FAS $S^{\prime}=S^{1} \cup S^{2}$ of $G^{\prime}$, with $\left|S^{1}\right| \leq\left|S^{2}\right|,\left|S^{\prime}\right|-\left|S_{o}^{\prime}\right|=\left|S^{\prime}\right|+\left|S_{o}\right|-2\left|S_{o}\right| \geq 2\left(\left|S^{\prime}\right|-\left|S_{o}\right|\right)$ so that $|S|-\left|S_{o}\right| \leq \frac{1}{2}\left(\left|S^{\prime}\right|-\left|S_{o}^{\prime}\right|\right)$. Thus, the two inequalities of $L$-reduction hold with $\alpha=1$ and $\beta=\frac{1}{2}$.

We now have the following:

Theorem 5.2. MIN-FAS- $k$ is APX-hard for all $k \geq 4$.

Proof. By Lemma 5.1, it is enough to show that MIN-FAS-4 is APX-hard. For this we show that MIN-VC-3 $\leq_{L}$ MIN-FAS-4.

We construct in polynomial time, from any 3-regular graph $G=(V, E)$ a 4-totalregular digraph $G^{\prime}=\left(V^{\prime}, A^{\prime}\right)$ where $V^{\prime}=\left\{v^{1}, v^{2} \mid v \in V\right\}$ and $A^{\prime}=\left\{\left(v^{1}, v^{2}\right) \mid v \in\right.$ $V\} \cup\left\{\left(u^{2}, v^{1}\right),\left(v^{2}, u^{1}\right) \mid\{u, v\} \in E\right\}$. Clearly, $G^{\prime}$ is a 4-total-regular digraph. For an example see Figure 3. For each FAS $F$ of $G^{\prime}$ we associate a VC $C$ of $G$ defined as $C=\left\{v \mid\right.$ either $\left(u^{2}, v^{1}\right) \in F$ or $\left.\left(v^{1}, v^{2}\right) \in F\right\}$. Further, $C$ is a VC of $G$ with $|C| \leq|F|$. For every edge $\{u, v\} \in E$, as $\left(u^{1}, u^{2}, v^{1}, v^{2}, u^{1}\right)$ is a dicycle in $G^{\prime}, F$ must contain at least one arc from this dicycle, and so, $C$ must contain either $u$ or $v$. Hence, $C$ is a VC of $G$, and by the construction of $C$ from $F,|C| \leq|F|$.

Next we show that if $F_{o}$ is a minimum FAS of $G^{\prime}$, then the associated VC $C_{o}$ of $G$ is a minimum VC of $G$ and $\left|F_{o}\right|=\left|C_{o}\right|$. As $F_{o}$ is a minimum FAS of $G$, without loss of generality we may assume that $F_{o}$ contains arcs only of the form $\left(v^{1}, v^{2}\right)$ for some $v \in V$. For, if $F_{o}$ contains arcs of the form $\left(u^{2}, v^{1}\right)$, then $F_{o}^{\prime}$ obtained from $F$ by replacing any such arc $\left(u^{2}, v^{1}\right)$ by $\left(v^{1}, v^{2}\right)$, we have $F_{o}^{\prime}$ an FAS of $G^{\prime}$ with $\left|F_{o}^{\prime}\right| \leq\left|F_{o}\right|$. Hence, $C_{o}=\left\{v \mid\left(v^{1}, v^{2}\right) \in F_{o}\right\}$. If $C_{o}$ is not a minimum $\mathrm{VC}$ of $G$, let $C$ be a minimum $\mathrm{VC}$ of $G$. Now $F=\left\{\left(v^{1}, v^{2}\right) \mid v \in C\right\}$ is an FAS of $G^{\prime}$. If not, let $K$ be a cycle in $G^{\prime}$ without containing any arc of $F$. Let $\left(u^{2}, v^{1}\right)$ be an arc in $K$. Then both $\left(v^{1}, v^{2}\right)$ and $\left(u^{1}, u^{2}\right)$ are in $K$. Hence, $u, v \notin C$, but $\{u, v\} \in E$ as $\left(u^{2}, v^{1}\right) \in K \subseteq A^{\prime}$. This implies that $C$ is not a $\mathrm{VC}$ of $G$, which contradicts our assumption. Hence $F$ must be an FAS of $G^{\prime}$. But then $|F|=|C|<\left|C_{o}\right|=\left|F_{o}\right|$, which contradicts that $F_{o}$ is a minimum FAS of $G^{\prime}$. Hence, $C_{o}$ is a minimum VC of $G$.

Also, $\left|F_{o}\right|=\left|C_{o}\right|$ and for any FAS $F$ of $G^{\prime},|C|-\left|C_{o}\right| \leq|F|-\left|F_{o}\right|$. So the transformation from $G$ to $G^{\prime}$ is an $L$-reduction with $\alpha=1$ and $\beta=1$.

Similarly, for MAX-SUBDAG, we first prove the following:

Lemma 5.3. $M A X-S U B D A G-k \leq_{L} M A X-S U B D A G-(k+1)$. 
Proof. From a $k$-total-regular digraph $G=(V, A)$ construct a $(k+1)$-total-regular digraph $G^{\prime}=\left(V^{\prime}, A^{\prime}\right)$ as constructed in the proof of Lemma 5.1. We shall use the notations used in the proof of Lemma 5.1 and let $B=\left\{\left(v^{1}, v^{2}\right) \mid v \in V\right\}$. Let $S=\left\{(u, v) \mid\left(u^{1}, v^{1}\right) \in S^{1}\right\}$ where without loss of generality we assume that $S^{\prime}=$ $S^{1} \cup S^{2} \cup B$ and $\left|S^{1}\right| \geq\left|S^{2}\right|$ and $\left(V^{i}, S^{i}\right)$ is a maximal SUBDAG of $G^{i}$, for $i=1,2$. Clearly, $(V, S)$ is a maximal SUBDAG of $G$. Now, if $S_{o}^{\prime}$ is a maximum SUBDAG of $G^{\prime}, S_{o}^{\prime}=S_{o}^{1} \cup S_{o}^{2} \cup B$, then note that $\left|S_{o}^{1}\right|=\left|S_{o}^{2}\right|$ and $S_{o}=\left\{(u, v) \mid\left(u^{1}, v^{1}\right) \in S_{o}^{1}\right\}$ is a maximum SUBDAG of $G$. Also, $\left|S_{o}^{\prime}\right|=2\left|S_{o}\right|+|B|=2\left|S_{o}\right|+n \leq 2\left|S_{o}\right|+|A| \leq 4\left|S_{o}\right|$, since $|A| \leq 2\left|S_{o}\right|$. So, the first inequality of $L$-reduction holds with $\alpha=4$. Next for any maximal SUBDAG $\left(V^{\prime}, S^{\prime}\right)$ of $G^{\prime},\left|S_{o}^{\prime}\right|-\left|S^{\prime}\right|=\left|S_{o}^{1}\right|+\left|S_{o}^{2}\right|+|B|-\left|S^{1}\right|-$ $\left|S^{2}\right|-|B|=\left|S_{o}^{1}\right|+\left|S_{o}^{2}\right|-\left|S^{1}\right|-\left|S^{2}\right| \geq\left|S_{o}\right|-|S|$. So, the second inequality of $L$-reduction holds with $\beta=1$.

We now prove the following:

Theorem 5.4. $M A X-S U B D A G$ - $k$ is $A P X$-complete for any $k \geq 4$.

Proof. By Lemma 5.3, it is enough to show that MAX-SUBDAG-4 is APX-hard. For this we show that MIN-VC-3 $\leq_{L}$ MAX-SUBDAG-4.

Let $G=(V, E)$ be any 3-regular graph. From $G$ we construct a 4 -total-regular digraph $G^{\prime}=\left(V^{\prime}, A^{\prime}\right)$ as constructed in Theorem 5.2. To each SUBDAG $\left(V^{\prime}, S^{\prime}\right)$ of $G^{\prime}$, we associate a VC of $G, C$ defined as $C=\left\{v \mid\right.$ either $\left(u^{2}, v^{1}\right) \in A^{\prime}-S^{\prime}$ or $\left.\left(v^{1}, v^{2}\right) \in A^{\prime}-S^{\prime}\right\}$. $C$ is indeed a VC of $G$. If $C$ is not then there exists $\{u, v\} \in E$ for which both $u$ and $v$ are not in $C$. For this edge $\{u, v\}, G^{\prime}$ contains the cycle $\left\{\left(u^{2}, v^{1}\right),\left(v^{1}, v^{2}\right),\left(v^{2}, u^{1}\right),\left(u^{1}, u^{2}\right)\right\}$. As none of $u$ and $v$ is in $C, A^{\prime}-S^{\prime}$ does not contain any of the arcs in this cycle. So, $A^{\prime}-S^{\prime}$ is not an FAS of $G^{\prime}$, which is a contradiction. Further, note that $\left|A^{\prime}\right|=2|E|+n,|C|<\left|A^{\prime}-S^{\prime}\right|=2|E|+n-\left|S^{\prime}\right|$ which implies that $\left|S^{\prime}\right| \leq 2|E|+n-|C|$.

Now, if $\left(V^{\prime}, S_{o}^{\prime}\right)$ is a maximum SUBDAG of $G^{\prime}$, then the $\mathrm{VC} C_{o}$ of $G$ associated with it is a minimum $\mathrm{VC}$ of $G$ and $\left|S_{o}^{\prime}\right|=2|E|+n-\left|C_{o}\right|$. To see this, note that $A^{\prime}-S_{o}^{\prime}$ is a minimum FAS of $G^{\prime}$. So by the proof of Theorem 5.2, $C_{o}$ is a minimum $\mathrm{VC}$ of $G$ and $\left|C_{o}\right|=\left|A-S_{o}^{\prime}\right|=2|E|+n-\left|S_{o}^{\prime}\right|$.

Next note that, any $\mathrm{VC} C$ in a 3 -regular graph $G$ contains at least $\frac{n}{2}$ vertices (see proof of Th. 4.2). Now, $\left|S_{o}^{\prime}\right|=2|E|+n-\left|C_{o}\right|=3 n+n-\left|C_{o}\right|=4 n-\left|C_{o}\right| \leq$ $8\left|C_{o}\right|-\left|C_{o}\right|=7\left|C_{o}\right|$. So the first inequality of $L$-reduction from $G$ to $G^{\prime}$ holds with $\alpha=7$.

Next, for any SUBDAG $\left(V^{\prime}, S^{\prime}\right)$ of $G^{\prime},\left|S_{o}^{\prime}\right|-\left|S^{\prime}\right|=\left|A^{\prime}\right|-\left|S^{\prime}\right|-\left|A^{\prime}\right|+\left|S_{o}^{\prime}\right|=$ $\left|A^{\prime}-S^{\prime}\right|-\left|A^{\prime}-S_{o}^{\prime}\right| \geq|C|-\left|C_{o}\right|$. Hence the second inequality of $L$-reduction holds with $\beta=1$.

Regarding MIN-MAX-SUBDAG, we have the following easy theorem:

Theorem 5.5. MIN-MAX-SUBDAG- $\leq 12$ is APX-hard.

Proof. In the proof of Theorem 3.1, we constructed an instance $G^{\prime}$ of MIN-MAXSUBDAG from an instance $G$ of MAX-SUBDAG in such a way that if $G$ is 4 total-regular then, every vertex in $G^{\prime}$ is of total degree at most 12 . Since MAXSUBDAG-4 is APX-complete, the result follows. 
Finally, we have:

Theorem 5.6. MAX-MIN-FVS- $\leq 6$, for digraphs, is APX-hard.

Proof. In the proof of Theorem 3.10, we constructed an instance $G^{\prime}$ of MAX-MINFVS, for digraphs, from an instance of $G$ of MAX-MIN-VC in such a way that if $G$ is of degree at most 5 , then $G^{\prime}$ is of total-degree at most 6. Since MAX-MIN$\mathrm{VC}-\leq 5$ is APX-complete it follows that MAX-MIN-FVS- $\leq 6$ is APX-hard.

\section{Concluding Remarks}

In this paper we have established hardness results for several NP-optimization problems related to MINLOP. These problems are variations or generalizations of well-known NP-optimization problems on graphs/digraphs. While for MAX-MINVC and MAX-MIN-FVS we have established strong results like those of Håstad [18] concerning MAX-IS and MAX-CLIQUE, for others we have just shown them to be APX-hard. Whether strong results about hardness of approximating such problems can be obtained is worth investigating. Despite such negative results, efforts may be made to obtain useful positive results giving efficient algorithms which may be $f(n)$-approximate for suitable function $f(n)$. Also, we do not have any results about MAX-MIN-FAS problem similar to MAX-MIN-FVS. These and other relevant issues concerning these problems are being pursued.

Acknowledgements. The authors thank C.R. Subramanian for a careful reading of an earlier draft and the anonymous referees for their comments and criticisms on [32] and the earlier version of this paper.

\section{REFERENCES}

[1] P. Alimonti and V. Kann, Hardness of approximating problems on cubic graphs, in Proc. 3rd Italian Conf. on Algorithms and Complexity. Springer-Verlag, Lecture Notes in Comput. Sci. 1203 (1997) 288-298.

[2] G. Ausiello, P. Crescenzi and M. Protasi, Fundamental Study: Approximate solution of NP optimization problems. Theoret. Comput. Sci. 150 (1995) 1-55.

[3] G. Ausiello, P. Crescenzi, G. Gambosi, V. Kann, A. Marchetti-Spaccamela and M. Protasi, Complexity and Approximation: Combinatorial Optimization Problems and Their Approximability Properties. Springer-Verlag, Berlin Heidelberg (1999).

[4] V. Bafna, P. Berman and T. Fujito, Constant ratio approximations of feedback vertex sets in weighted undirected graphs, in 6th Annual International Symposium on Algorithms and Computation (1995).

[5] A. Brandstädt, V.D. Chepoi and F.F. Dragan, The algorithmic use of hypertree structure and maximum neighborhood orderings. Discrete Appl. Math. 82 (1998) 43-77. 
[6] A. Brandstädt and D. Kratsch, On domination problems for permutation and other graphs. Theoret. Comput. Sci. 54 (1987) 181-198.

[7] S. Chanas and P. Kobylański, A new heuristic algorithm solving the linear ordering problem. Comput. Optim. Appl. 6 (1996) 191-205.

[8] M.S. Chang, Efficient algorithms for the domination problems on interval and circular-arc graphs. SIAM J. Comput. 27 (1998) 1671-1694.

[9] A. Chaudhary and S. Vishwanathan, Approximation algorithms for achromatic number, in Proc. 8th Ann. ACM-SIAM Symp. on Discrete Algorithms. ACM-SIAM (1997) 558-563.

[10] G.A. Cheston, G. Fricke, S.T. Hedetniemi and D.P. Jacobs, On the computational complexity of upper fractional domination. Discrete Appl. Math. 27 (1990) 195-207.

[11] P. Crescenzi, V. Kann, R. Silvestri and L. Trevisan, Structures in approximation classes, in 1st. Annu. Int. Conf. on Computing and Combinatorics. Springer-Verlag, Lecture Notes in Comput. Sci. 959 (1995) 539-548.

[12] U. Feige and J. Kilian, Zero knowledge and the chromatic number. Proc. Comp. Complexity (1996).

[13] M. Fraber, Independent domination in chordal graphs. Oper. Res. Lett. 1 (1982) 134-138.

[14] M. Fraber and J.M. Keil, Domination in permutation graphs. J. Algorithms 6 (1985) 309321.

[15] T. Fujito, Personal communication (1999).

[16] M. Grötschel, M. Jünger and G. Reinelt, A cutting plane algorithm for linear ordering problem. Oper. Res. 32 (1984) 1195-1220.

[17] M. Grötschel, M. Jünger and G. Reinelt, On the acyclic subgraph polytope. Math. Programming 33 (1985) 28-42.

[18] J. Håstad, Clique is hard to approximate within $n^{1-\epsilon}$, in Proc. 37th IEEE Sympo. on Foundation of Comput. Sci. (1996) 627-636.

[19] F. Harary, Graph Theory. Addition-Wesley, Reading, MA (1969).

[20] F. Harary, Maximum versus minimum invariants for graphs. J. Graph Theory 7 (1983) $275-284$.

[21] F. Harary and S. Hedetniemi, The achromatic number of a graph. J. Combin. Theory 8 (1970) 154-161.

[22] M.M. Halldórsson, Approximating the minimum maximal independence number. Inform. Process. Lett. 46 (1993) 169-172.

[23] R.W. Irving, On approximating the minimum independent dominating set. Inform. Process. Lett. 37 (1991) 197-200.

[24] V. Kann, On the Approximability of NP-complete Optimization Problems, Ph.D. Thesis. Department of Numerical Analysis and Computing Science, Royal Institute of Technology, Stockholm (1992).

[25] V. Kann, Polynomially bounded minimization problems that are hard to approximate. Nordic J. Comput. 1 (1994) 317-331.

[26] S. Khanna, R. Motwani, M. Sudan and U. Vazirani, On syntactic versus computational views of approximability, in Proc. 35th Ann. IEEE Symp. on Foundations of Computer Science (1994) 819-836.

[27] C. Lund and M. Yannakakis, On the hardness of approximating minimization problems. $J$. ACM 41 (1994) 960-981.

[28] C.H. Papadimitriou and M. Yannakakis, Optimization, Approximation, and Complexity Classes. J. Comput. System Sci. 43 (1991) 425-440.

[29] K. Peters, R. Laskar and S.T. Hedetniemi, Maximinimal/Minimaximal connectivity in graphs. Ars Combinatoria 21 (1986) 59-70.

[30] D.F. Manlove, Minimaximal and maximinimal optimization problems: A partial order-based approach, Ph.D. Thesis. University of Glasgow (1998).

[31] S. Mishra and K. Sikdar, On approximation solutions of linear ordering and related NPOptimization problems on graphs (Extended Abstract), Electronic Notes in Discrete Mathematics, Vol. 8, edited by H. Broersma, U. Faigle, J. Hurink and S. Pickl. Elsevier Science Publishers (2001), Full version submitted for publication. 
[32] S. Mishra and K. Sikdar, On the hardness of approximating some NP-optimization problems related to minimum linear ordering problem (extended abstract), edited by J. van Leeuwen et al., IFIP TCS 2000. Lecture Notes in Comput. Sci. 1872 (2000) 186-199.

[33] S. Ueno, Y. Kajtani and S. Gotoh, On the nonseparating independent set problem and feedback set problem for graphs with no vertex exceeding three. Discrete Math. 72 (1988) 355-360.

Communicated by J. Gruska.

Received February 14, 2001. Accepted August 24, 2001. 